\title{
Anaplastic Ganglioglioma
}

National Cancer Institute

\section{Source}

National Cancer Institute. Anaplastic Ganglioglioma. NCI Thesaurus. Code C4717.

A WHO grade III neuroepithelial neoplasm composed of neoplastic, mature gang lion cells and anaplastic glial cells. The anaplastic changes in the glial component and high MIB-1 and TP53 labeling indices may indicate aggressive behavior. However, the correlation of histological anaplasia with clinical outcome is inconsistent. (Adapted from WHO) 